\title{
Endoscopic resection of an esophageal duplication cyst with spraying of anhydrous alcohol
}

A 56-year-old man presented with progressive dysphagia for 12 months, but reported no weight loss and no significant medical history. Gastroscopy revealed a bulge with normal overlying mucosa in the lower esophagus ( $\bullet$ Fig. 1). Endoscopic ultrasound (EUS) showed a $5-\mathrm{cm}$ hypoechoic intraluminal cystic mass with clear margins originating from the muscularis propria ( $\mathbf{F i g . 2}$ ). A well-defined cystic mass without internal enhancement was observed on computed tomography (CT) ( $\bullet$ Fig. 3).

Endoscopic submucosal dissection (ESD) of the esophageal lesion was performed ( Video 1). The lesion was raised by submucosal injection of $100 \mathrm{~mL}$ glycerol fructose $(10 \%)$ mixed with $1 \mathrm{~mL}$ of indigo carmine. After an incision had been made on the cyst wall with a dual-knife (KD-650L; Olympus, Tokyo, Japan), the yellowish fluid of the cyst drained gradually and completely into the esophageal lumen. The lesion was completely and uneventfully resected using the dual-knife and a hook-knife. Good visualization of the cavity of the cystic lesion was subsequently obtained, and anhydrous alcohol was sprayed into the cavity using a spray tube. There were no complications such as bleeding or perforation.

A diagnosis of an esophageal duplication cyst was made by histopathological examination of the resected specimen. A repeat endoscopy was performed 7 days later, and anhydrous alcohol was sprayed once again ( $\bullet$ Fig.4). The patient reported a rapid recovery and denied dysphagia during a follow-up period of 12 months.

Esophageal duplication cysts are rare congenital anomalies of the foregut and the majority of patients are asymptomatic [1]. Surgical resection is generally required for cysts that cause symptoms such as dysphagia, epigastric pain, and retrosternal pain [2]. Endoscopic treatment of esophageal duplication cysts has increased in recent years because of its

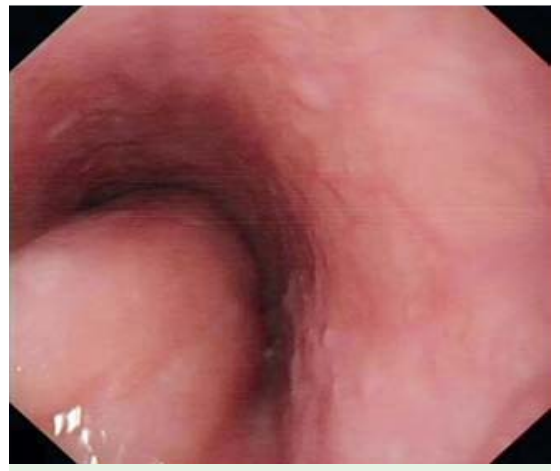

Fig. 1 Endoscopic image showing a bulge in the lower esophageal wall with normal overlying mucosa that was later shown to be an esophageal duplication cyst.

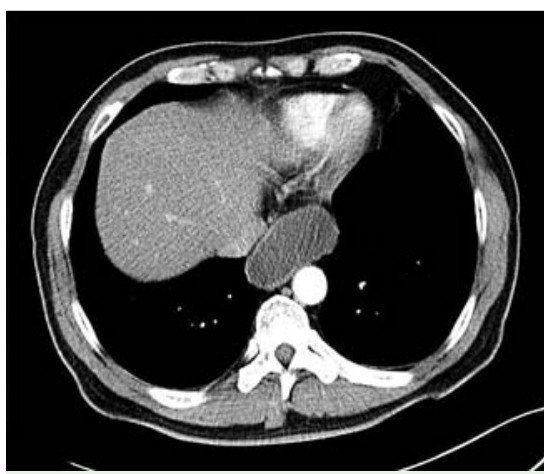

Fig. 3 Computed tomography (CT) scan image showing a cystic mass without internal enhancement in the lower esophagus.

minimal invasiveness $[3,4]$. In this case, after performing ESD, we sprayed anhydrous alcohol, which is usually used for treating liver or ovarian cysts. Because anhydrous alcohol can destroy the epithelial cell layer of the cyst wall, the recurrence rate of cysts may, theoretically, be lower. Further studies on the long-term efficacy and safety of this treatment are required.

Endoscopy_UCTN_Code_TTT_1AO_2AD

Competing interests: None

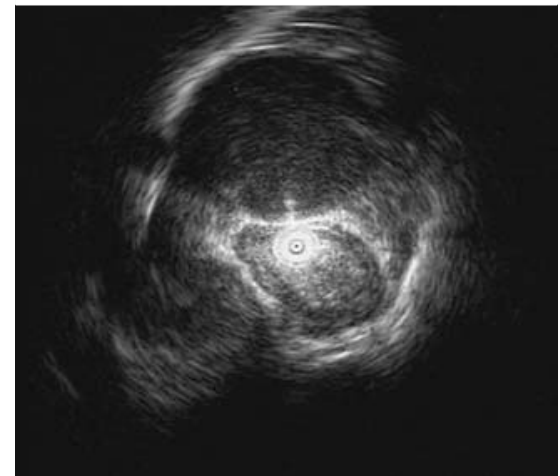

Fig. 2 Endoscopic ultrasound (EUS) view of the lesion showing a 5-cm hypoechoic cystic mass with clear margins originating from the muscularis propria.

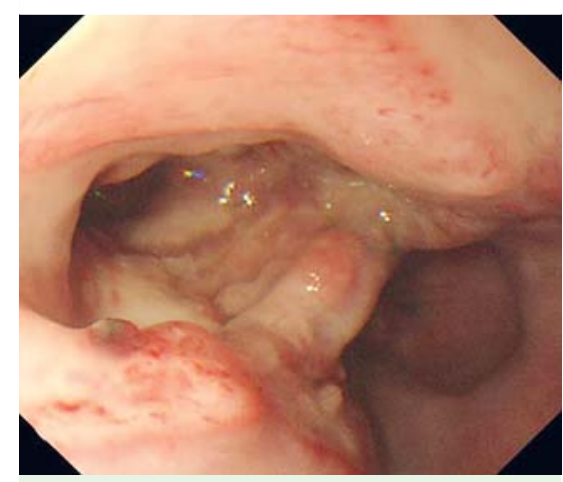

Fig.4 Appearance at follow-up endoscopy 7 days after endoscopic submucosal dissection (ESD) of an esophageal duplication cyst and spraying of anhydrous alcohol.

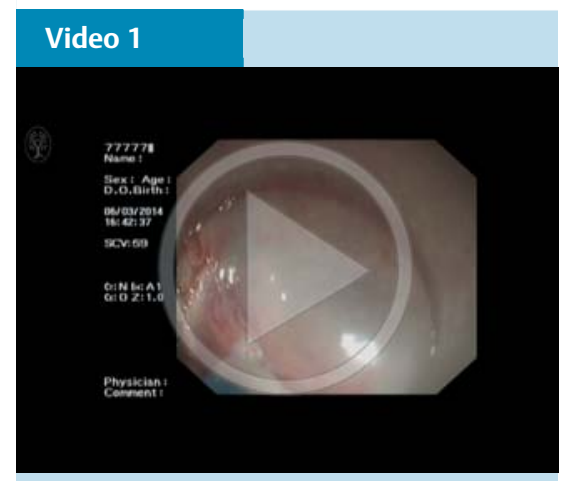

Endoscopic submucosal dissection (ESD) of the esophageal lesion. 
Yi Mou', Dingke Wen², Qin Liu', Honglin Chen ${ }^{1}$, Hang Yi ${ }^{1}$, Wei Liu', Bing $\mathrm{Hu}^{1}$

${ }^{1}$ Department of Gastroenterology, West China Hospital, Chengdu, Sichuan, China

${ }^{2}$ West China School of Medicine, Sichuan University, Chengdu, Sichuan, China

\section{References}

1 Turkyilmaz A, Eroglu A, Subasi M et al. Intramural esophageal bronchogenic cysts: a review of the literature. Dis Esophagus 2007; 20: $461-465$

2 Singh S, Lal P, Sikora SS et al. Squamous cell carcinoma arising from a congenital duplication cyst of the esophagus in a young adult. Dis Esophagus 2001; 14: 258

3 Joyce AM, Zhang PJ, Kochman ML et al. Complete endoscopic resection of an esophageal duplication cyst (with video). Gastrointest Endosc 2006; 64: 288-289

4 Tang $X$, Jiang $B$, Gong $W$ et al. Endoscopic submucosal tunnel dissection of a bronchogenic esophageal cyst. Endoscopy 2014; 46: E626-E627

\section{Bibliography}

Dol http://dx.doi.org/

10.1055/s-0034-1392502

Endoscopy 2015; 47: E348-E349

(c) Georg Thieme Verlag KG

Stuttgart · New York

ISSN 0013-726X

\section{Corresponding author}

\section{Bing Hu, MD}

Department of Gastroenterology West China Hospital, Sichuan University No. 37 Guo Xue Xiang

Chengdu

Sichuan, 610041

P.R. China

hubingnj@163.com 